\title{
PSEUDOCOMPLEMENTED DISTRIBUTIVE LATTICES WITH SMALL ENDOMORPHISM MONOIDS
}

\author{
M.E. Adams, V. Koubek and J. Sichler
}

By a result of K.B. Lee, the lattice of varieties of pseudocomplemented distributive lattices is the $\omega+1$ chain

$$
B_{-1} \subset B_{0} \subset B_{1} \subset \ldots \subset B_{n} \subset \ldots \subset B_{\omega}
$$

where $B_{-1}, B_{0}, B_{1}$ are the varieties formed by all trivial, Boolean, and Stone algebras, respectively. General theorems on relative universality proved in the present paper imply that there is a proper class of non-isomorphic algebras in $B_{3}$ with finite endomorphism monoids, while every infinite algebra from $B_{2}$ has infinitely many endomorphisms. The variety $B_{4}$ contains a proper class of non-isomorphic algebras with endomorphism monoids consisting of the identity and finitely many right zeros; on the other hand, any algebra in $B_{3}$ with a finite endomorphism monoid of this type must be finite.

\section{Introduction}

For an arbitrary algebra $L$, let $\operatorname{End}(L)$ denote the monoid of all endomorphisms of $L$.

Received 6 July 1983. The support of NSERC is gratefully acknowledged by $V$. Koubek and J. Sichler.

Copyright Clearance Centre, Inc. Serial-fee code: 0004-9727/83 $\$ A 2.00+0.00$. 
A pseudocomplemented distributive lattice is an algebra

$\left(L ; \vee, \wedge,{ }^{*}, 0,1\right)$ of type $(2,2,1,0,0)$ where $(L ; \vee, \wedge, 0,1)$ is a distributive $(0,1)$-lattice and the unary operation * of pseudocomplementation is defined by $y \leq x^{*}$ if and only if $x \wedge y=0$ in $(L ; \vee, \wedge, 0,1)$. Pseudocomplemented distributive lattices form a variety $B_{\omega}$, Ribenboim [18], and the lattice of its subvarieties is an $\omega+1$ chain $B_{-1} \subset B_{0} \subset B_{1} \subset B_{2} \subset \ldots \subset B_{\omega}$, Lee [11]. Of these varieties, two have already been studied extensively: the variety $B_{0}$ of Boolean algebras and the variety $B_{1}$ of stone algebras $B_{-1}$ is the trivial variety). Further information and references can be found in, for example, Balbes and Dwinger [3] or Grätzer [5], [6].

Independently, Magill [12] and Schein [19] have shown that a Boolean algebra is uniquely determined by its endomorphism monoid: for $K, L \in B_{0}, \operatorname{End}(K) \cong \operatorname{End}(L)$ only if $K \cong L$. Investigations of other varieties of pseudocomplemented distributive lattices [1] have extended this result to the variety $B_{1}$ of stone algebras. For $B_{2}$ the situation is somewhat different. If $\operatorname{End}(K) \cong \operatorname{End}(L)$ for nonisomorphic $K, L \in B_{2}$, then there is a uniquely defined algebra $L^{+} \cong K$; that is, an algebra in $B_{2}$ is determined by its endomorphism monoid up to one of two algebras. For $B_{3}$ and larger varieties a radical change occurs: for any infinite cardinal $\kappa$ there exists a family $\left[L_{i} \in B_{3}: i<2^{k}\right\}$ of pair-wise nonisomorphic algebras with $\left|L_{i}\right|=\kappa$ and $\operatorname{End}\left(L_{i}\right) \cong$ End $\left(L_{j}\right)$ for all $i, j \in 2^{k}$. This fact is an immediate consequence of the more general result [1] that $B_{3}$ is an almost universal variety. A category $C$ is almost zoniversal if the category $G$ of (undirected) graphs and all their compatible maps is isomorphic to a subcategory of $C$ formed by all nonconstant morphisms between objects from a suitably chosen subclass $D$ of $C$; note that this definition requires that the nonconstant morphisms between members of $D$ be closed under composition.

For any minimal prime ideal $I \subseteq K \in B_{\omega}$, the mapping $H: K+\{0,1\}$ given by $h^{-1}\{0\}=I$ is a homomorphism. A homomorphism $g: K \rightarrow L$ in $B_{\omega}$ 
is constant if and only if $g(K)=\{0,1\}$, that is if $g(K)$ is the set of constants of $L$. Since every pseudocomplemented distributive lattice has as least one minimal prime ideal, there exist constant homomorphisms between any two such algebras. The constant endomorphisms are precisely the right zeros in $\operatorname{End}(K)$ and thus every nontrivial $K \in B_{\omega}$ has at least one right zero in its endomorphism monoid [1].

All infinite algebras of cardinality $K$ constructed to prove the almost universality of $B_{3}$ in [1] have $2^{k}$ right zero endomorphisms; therefore no light is shed on the question of whether an infinite pseudocomplemented distributive lattice necessarily has an infinite endomorphism monoid. Although this is always the case in $B_{2}$, a surprising reversal occurs in larger varieties; Corollary 1.3 below claims the existence of arbitrarily large algebras in $B_{3}$ that have only finitely many endomorphisms. Furthermore, it will be seen that the construction presented in [1] was no accident: if $K \in B_{3}$ is infinite then either $K$ has infinitely many constant endomorphisms or there exists a non-constant nontrivial endomorphism of $K$ (see Theorem 1.1). By contrast, $B_{4}$ contains arbitrarily large algebras whose endomorphism monoids consist of the identity mapping and finitely many right zeros (Corollary 1.5). The results proved here will be more general, however, and additional concepts are needed for their formulation.

A variety $V$ is $W$-coniversal if $W$ is a subvariety of $V$ and if the category of all compatible maps between (undirected) graphs is isomorphic to a subcategory $C$ of $V$ consisting of all those homomorphisms $h: C_{1}+C_{2}$ between objects of $c$ for which $h\left(c_{j}\right) \in V W$. If, in addition, there are less than $k$ homomorphisms $h: c_{1}+c_{2}$ with $h\left(C_{1}\right) \in W$ for any pair of objects in $C$, the variety $V$ is $(\kappa, W)-$ zoniversal. In this terminology, since $\{0,1\}$ is a Boolean algebra, the almost universality of $B_{3}$ shown in [1] implies that $B_{3}$ is $B_{0}$ universal. By the preceding discussion, however, [1] does not imply $\left(\kappa, B_{0}\right)$-universality for any cardinal $k$. The results presented here are as follows. 
THEOREM 1.1. $B_{3}$ is neither $\left(\omega, B_{0}\right)$-umiversal nor $\left(\omega, B_{1}\right)-$ wiversal.

THEOREM 1.2. $B_{3}$ is $\left(\omega, B_{2}\right)$-universal.

In fact, a stronger result is shown: $B_{3}$ is $\left(m, B_{2}\right)$-universal for some integer $m$.

In connection with the immediate consequence below of Theorem 1.2 (see, for instance, Pultr and Trnková [17]) it is also interesting to recall that every infinite Boolean algebra has an uncountable endomorphism monoid.

COROLLARY 1.3. In $B_{3}$, there exists a proper class of pairwise nonisomorphic algebras whose endomorphism monoids are finite.

THEOREM 1.4. $B_{n}$ is $\left(\omega, B_{0}\right)$-universal for every $n \geq 4$.

As before, it is actually shown that $B_{4}$ is $\left(m, B_{0}\right)$-universal for some finite $m$. Furthermore, if Boolean, the image of an endomorphism is always the subalgebra $\{0,1\}$; the statement below is an immediate consequence of this fact.

COROLLARY 1.5. In $B_{4}$, there exists a proper class of pairwise nonisomorphic algebras whose endomorphism monoids consist of the identity and finitely many right zeros.

\section{Preliminaries}

In Priestley [13], a topological duality was introduced for the category of distributive $(0,1)$-lattices. A brief outline follows; for further information see, for instance, Davey and Duffus [4] or Priestley [16].

A mapping $\psi: P_{1} \rightarrow P_{2}$ between partially ordered sets $P_{1}, P_{2}$ is order preserving if $x \leq y$ implies $\psi(x) \leq \psi(y)$. Let $\operatorname{Min}(P)$ denote the set of all minimal elements of a poset $P$. Further, for $S \subseteq P$, set $[S)=\{x: \exists s \in S, x \geq s\},(S]=\{x: \exists s \in S, x \leq s\}$, and $\operatorname{Min}(S)=\operatorname{Min}(P) \cap(S] \quad$ if $S=\{x\}$ then $\operatorname{Min}(S)$ is written as $\operatorname{Min}(x)$ ). A subset $S$ of $P$ is increasing if $[S)=S$, decreasing if $S=(S]$. A 
poset $P$ equipped with a topology is totally order disconnected if, for $x, y \in P, x \neq y$ implies the existence of a clopen decreasing set $S \subseteq P$ such that $x \in S$ and $y \notin S$.

PROPOSITION 2.1 (Priestley [13]). The category $D_{0,1}$ of all $(0,1)$-homomorphisms of distributive $(0,1)$-lattices is dually isomorphic to the category $T$ of all continuous order preserving maps of compact totally order disconnected spaces.

Under this duality the poset associated with a distributive $(0,1)-$ lattice is the inclusion ordered poset of its prime ideals, and lattice elements correspond to clopen decreasing subsets of the representing totally order disconnected space. Furthermore, if $L_{j}$ is a distributive $(0,1)$-lattice associated with the space $\left(P_{j}, \tau_{j}\right)$ for $j=1,2$, and if $h: L_{1} \rightarrow L_{2}$ is a $(0,1)$-homomorphism represented by the continuous order preserving map $\psi: P_{2} \rightarrow P_{1}$, then, for the clopen decreasing set $X \subseteq P_{1}$ corresponding to $x \in L_{1}$, the element $h(x)$ of $L_{2}$ corresponds to the clopen decreasing set $\psi^{-1}(X) \subseteq P_{1}$.

PROPOSITION 2.2 [2]. The category $G$ of graphs is dually isomorphic to a full subcategory of the category $T_{5}$ of all compact totally order disconnected spaces with five distinguished elements and all continuous order preserving maps that also preserve these five elements.

An object of $T_{5}$ is thus a compact totally order disconnected space $(P, \tau)$ together with five distinct elements $a_{i}$ of $P$ for $i<5$; as given in [2], these five elements are minimal in $P$ and the partial order $P$ is connected. Inverting the order and retaining the topology of every such space gives rise to a full subcategory of $T_{5}$ isomorphic to the original one; thus it can be assumed that $P$ is connected and that all five distinguished elements are maximal in the order of $P$.

Since every pseudocomplemented distributive lattice has 0 and 1 , Priestley's duality restricts to a topological duality for $B_{\omega}$. A totally order disconnected space $(P, \tau)$ has the p-property if $[S)$ is clopen for every clopen decreasing set $S \subseteq P$. An ord $\in$ r preserving map 
$\psi: P_{1} \rightarrow P_{2}$ is a $p$-map if $\psi(\operatorname{Min}(x))=\operatorname{Min}(\psi(x))$ for every $x \in P_{1}$. The category ${ }_{\omega}^{B}$ is dually isomorphic to the category $\mathbb{T}^{p}$ of all compact totally order disconnected spaces with the p-property and all continuous $p$-maps between such spaces. For more extensive background information see Priestiey [15]. Note that any constant homomorphism of pseudocomplemented distributive lattices corresponds in this duality to a constant $p$-map whose value is a minimal element.

Finally, for $n<\omega$, we need to recognize spaces with the p-property that represent algebras in $B_{n}$.

PROPOSITION 2.3 (Lee [11]). For $L \in B_{\omega}$ and $n \geq 1$, the algebra $L$ bezongs to $B_{n}$ if and only if every prime ideal of $L$ contains at most $n$ minimal prime ideals.

\section{Proof of Theorem 1.1}

In this section, let $L \in B_{3}$ be an infinite algebra with only a finite number of constant endomorphisms; if $(P, \tau)$ represents such $L$ then $\operatorname{Min}(P)$ must be finite. Denote $P(M)=\{x \in P: \operatorname{Min}(x)=M\}$ for every $M \subseteq \operatorname{Min}(P)$.

LEMMA 3.1. $P(M)$ is clopen for every $M \subseteq \operatorname{Min}(P)$.

Proof. Since $P$ is totally order disconnected, for any $m \in \operatorname{Min}(P)$ there exists a clopen decreasing set $Q_{m} \subseteq P$ such that $\operatorname{Min}\left(Q_{m}\right)=\{m\}$. By the $p$-property, $\left[Q_{m}\right]$ is clopen, so that

$$
P(M)=n\left(\left[Q_{m}\right): m \in M\right) \backslash \cup\left(\left[Q_{m}\right): m \in \operatorname{Min}(P) \backslash M\right)
$$

is clopen as well.

LEMMA 3.2. For every $p \in P(M) \backslash \operatorname{Min}(P)$ with $|M|<3$ there exists a non-trivial $h \in \operatorname{End}(L)$ whose image is represented by $\{p\} \cup \operatorname{Min}(p)$.

Proof. Suppose $M=\{m\}$ and $p \in P(M) \backslash \operatorname{Min}(P)$. There exists a clopen decreasing $Q \subseteq P$ not containing $p$ and such that $Q \supseteq \operatorname{Min}(P)$; set $\psi(x)=m$ for $x \in Q$ and $\psi(x)=p$ for $x \in P \backslash Q$. It is clear that $\psi$ is a $p$-map and that $\psi(P)=\{p\} \cup \operatorname{Min}(p)$. 
Let $M=\{m, n\}$ and $p \in P(M) \backslash \operatorname{Min}(P)$. Define $\psi: P \rightarrow P$ by $\psi(x)=n$ if $\operatorname{Min}(x)=\{n\}, \psi(x)=m$ for $n \neq \operatorname{Min}(x)$, and $\psi(x)=p$ otherwise. Then $\psi$ is a $p$-map whose continuity follows from Lemma 3.1, and again $\psi(P)=\{p\} \cup \operatorname{Min}(p)$.

PROPOSITION 3.3. End $(L)$ is infinite for arbitrary infinite $L \in B_{2} \cdot$

Proof. Recall that $\operatorname{Min}(P)$ is finite; Proposition 2.3 implies that $P(M)$ must be infinite for some $M \subseteq \operatorname{Min}(P)$ with $|M|<3$. Lemma 3.2 concludes the proof.

LEMMA 3.4. If $P(M)=\emptyset$ for all two-element $M \subseteq M i n(P)$ then End $(L)$ is infinite.

Proof. Since $\operatorname{Min}(P)$ is finite, for some $M \subseteq \operatorname{Min}(P)$ the clopen set $P(M)$ must be infinite. By the hypothesis and by Lemma 3.2 it is enough to assume that $|M|=3$; since $L \in B_{3}$, Proposition 2.3 implies that $P(M)$ is an increasing set. For each $p \in P(M)$ now define $\Psi_{p}$ by $\Psi_{p}(x)=p$ for $x \in P(M), \psi(x)=m$ whenever $\operatorname{Min}(x)=\{m\}$ for some $m \in M$, and $\psi_{p}(x)=x$ otherwise. From Lemma 3.1 it is clear that $\psi_{p}$ is a $p$-map for every $p \in P(M)$. Thus $\operatorname{End}(L)$ is infinite.

There exist arbitrarily large graphs with no non-trivial endomorphisms [17]. If $B_{3}$ were $\left(\omega, B_{j}\right)$-universal for some $j<2$ there would certainly exist an infinite algebra $L$ in $B_{3}$ with finite $\operatorname{End}(L)$ and such that $h(L) \in B_{2}{ }^{B} j$ for no $h \in \operatorname{End}(L)$. The two preceding lemmata show that this is impossible.

\section{Proof of Theorem 1.2}

A graph is a pair $(X, R)$ where $R$ is a set of two-element subsets of $X$. For graphs $\left(X_{1}, R_{1}\right),\left(X_{2}, R_{2}\right)$ a mapping $\varphi: X_{1}+X_{2}$ is compatible if $\{\varphi(x), \varphi(y)\} \in R_{2}$ for every $\{x, y\} \in R_{1}$.

By Proposition 2.2, there is a full and faithful contravariant functor $\Phi$ from the category $G$ of all graphs and their compatible maps to the category $T_{5}$ of all compact totally order disconnected spaces with five 
distinguished elements and continuous order preserving maps that preserve these elements: the existence of $\Phi$ shows that $G$ is dually isomorphic to a full subcategory of $T_{5}$. The image $(P, \tau)=\Phi(X, R)$ of any $(X, R) \in G$ is a compact totally order disconnected space in which $P$ is order connected and the five constants $a_{i}$ for $i<5$ are maximal elements of $P$. To show that $B_{3}$ is $\left(\omega, B_{2}\right)$-universal, a faithful contravariant functor $\Psi: G \rightarrow T^{p}$ containing $\Phi$ will be constructed in such a way that $\Psi(X, R)$ is the space of an algebra in $B_{3}$ and, if $\psi: \Psi\left(X_{1}, R_{1}\right) \rightarrow \Psi\left(X_{2}, R_{2}\right)$ is a morphism in $T^{p}$ then either $\psi=\Psi(\varphi)$ for some compatible $\varphi:\left(X_{2}, R_{2}\right) \rightarrow\left(X_{1}, R_{1}\right)$ or $\psi$ is one of finitely many mappings with $\psi\left(\Psi\left(X_{1}, R_{1}\right)\right)$ representing an algebra in $B_{2}$.

A graph $G$ is rigid if the only compatible map of $G$ into itself is the identity. Once and for all select a finite rigid graph $(V, E)$ with $|V|>7$; such graphs exist according to Hedrlín and Pultr [9] (see also [17]). Set $V=\left\{v_{i}: i<|V|\right\}$, and let $T$ denote the collection of all three-element subsets of $V$. For convenience, denote $t_{i}=\left\{v_{i}, v_{6}, v_{7}\right\}$ for all $i<6$, and let $c=\left\{c_{i}: i<5\right\}$.

The functor $\Psi$ is defined as follows.

For $(X, R) \in G$, let

$$
Q=V \cup E \cup C \cup\{b\} \cup\left(T \backslash\left\{t_{5}\right\}\right) \cup P \text {, }
$$

where $(P, \tau)=\Phi(X, R)$ and the union is disjoint; intuitively, the triple $t_{5}$ will be replaced by a copy of $P$. Define a partial order on $Q$ by

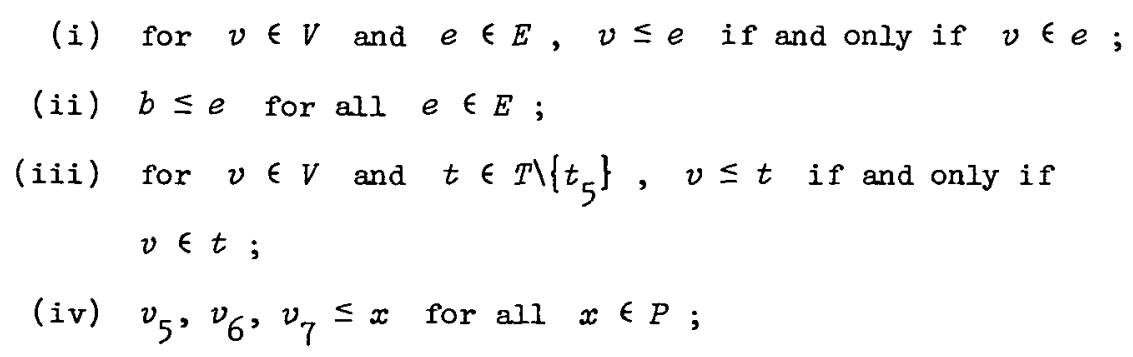


(v) if $i<5$ then $v_{6}, v_{7} \leq c_{i} \leq a_{i}, t_{i}$ for $c_{i} \in C$ and for the distinguished maximal element $a_{i}$ of $P$;

(vi) for $x, y \in P, x \leq y$ in $Q$ if and only if $x \leq y$ in $P$.

Let $\sigma$ denote the topology on $Q$ obtained as the union of $\tau$ on $P$ with the discrete topology on the finite set $Q \backslash P$; the space $(Q, \sigma)=\Psi(X, R)$ clearly is compact and totally disconnected.

For any morphism $\varphi:\left(X_{2}, R_{2}\right) \rightarrow\left(X_{1}, R_{1}\right)$ in $G$, let $\Psi(\varphi): \Psi\left(X_{1}, R_{1}\right)+\Psi\left(X_{2}, R_{2}\right)$ be defined as the extension of $\Phi(\varphi)$ by the identity mapping on $Q_{1} \backslash P_{1}=Q_{2} \backslash P_{2}$. It is routine to verify that $\Psi(\varphi)$ is a continuous order preserving mapping. Since $\Phi(X, R)$ is a totally order disconnected space, it follows easily that $(Q, \sigma)$ also is such a space. Clearly $[S]=[\operatorname{Min}(S))$ for any clopen decreasing set $S \subseteq Q$; hence as $\operatorname{Min}(Q)=V \cup\{b\}$, the set $[S)$ is either a finite subset of $Q \backslash P$ or $[S\rangle$ contains $P$. In either case $[S)$ is clopen, so that $\Psi(X, R)$ has the $p$-property. Since $|\operatorname{Min}(q)| \leq 3$ for all $q \in Q$, Proposition 2.3 shows that $\Psi(X, R)$ represents an algebra in $B_{3}$. The mapping $\Psi(\psi)$ is the identity on $Q \backslash P \supseteq \operatorname{Min}(Q)$, and it is readily apparent that $\Psi(\varphi)$ is a p-map. This concludes the proof of the claim below.

LEMMA 4.1. $\Psi: G \rightarrow T^{p}$ is a well defined contravariant faithful fronctor.

Observe that, for $j=1,2$, if $L_{j} \in B_{\omega}$ is represented by $\left(Q_{j}, \sigma_{j}\right)$ and $\psi: Q_{1}+Q_{2}$ is a morphism in $T^{p}$ representing $h: L_{2} \rightarrow L_{1}$, then $h\left(L_{2}\right) \in B_{n}$ if and only if the subspace $\psi\left(Q_{1}\right) \subseteq Q_{2}$ with the order induced by $Q_{2}$ represents an algebra in $B_{n}$.

Thus it must be shown that, for $\left(X_{j}, R_{j}\right) \in G$, there are only finitely many continuous order preserving p-maps $\psi: \Psi\left(X_{1}, R_{1}\right) \rightarrow \Psi\left(X_{2}, R_{2}\right)$ with $\psi\left(Q_{1}\right)$ representing algebras from $B_{2}$, and that any other $\psi$ has the form $\psi=\Psi(\varphi)$ for some $G$-morphism $\varphi:\left(X_{2}, R_{2}\right) \rightarrow\left(X_{1}, R_{1}\right)$. 
For the remainder of this section let $\psi: \Psi\left(X_{1}, R_{1}\right) \rightarrow \Psi\left(X_{2}, R_{2}\right)$ be a continuous order preserving p-map with $\left(x_{j}, R_{j}\right) \in G$.

LEMMA 4.2. If $\psi \wedge(V \cup\{b\})$ is not one-to-one then $\psi\left(Q_{1}\right) \subseteq V \cup\{b\} \cup C$, and $\psi \wedge P_{1}$ is constant.

Proof. Recall that $V \cup\{b\}=\operatorname{Min}(Q) ;$ furthermore, $|\operatorname{Min}(c)|=2$ for $c \in C$, and $|\operatorname{Min}(x)|=3$ for all $q \in Q \backslash(\operatorname{Min}(Q) \cup C)$.

Suppose that $\psi(u)=\psi(v)$ for distinct $u, v \in V$; then $\psi(u)=m$ is minimal since $\psi$ is a p-map.

Consider the case of $\psi(u) \notin\left\{v_{6}, v_{7}\right\}$ first. For every $w \in V \backslash\{u, v\}$ there exists $t \in T$ with $\operatorname{Min}(t)=\{u, v, w\}$, and $\operatorname{Min}(\psi(t))=\psi(\operatorname{Min}(t))$ implies that $\psi(t)=\psi(w)=m$. For every three-element subset $U$ of $V \subseteq \operatorname{Min}(Q)$ there exists $t \in T$ with $\operatorname{Min}(t)=U$, so that both $\psi(V)=\{m\}$ and $\psi\left(\left(T \backslash\left\{t_{5}\right\}\right) \cup P_{1} \cup C\right)=\{m\}$ easily follow. If $\{u, v\} \in E$ then $\psi(\{b, u, v\})=\{m\}$ is similarly obtained from $\psi(V)=\{m\}$, and hence $\psi$ is a constant p-map.

Assume next that $m \in\left\{v_{6}, v_{7}\right\}$; if $\operatorname{Min}(t)=\{u, v, w\}$ then either $\psi(t)=\psi(w)=m$, or $\psi(t) \in C$ and $m \neq \psi(w) \in\left\{v_{6}, v_{7}\right\}$. If $\psi(V)=\{m\}$ then $\psi\left(P_{1} \cup\left(T \backslash\left\{t_{5}\right\}\right)\right)=\{m\}$ and $\psi(E) \subseteq C \cup \vee$ finish the proof. If $\psi(V)=\left\{v_{6}, v_{7}\right\}$ then either $\psi\left(\left\{v_{5}, v_{6}, v_{7}\right\}\right)=m$ or $\left\{v_{6}, v_{7}\right\}$; in the first case $\psi\left(P_{1}\right)=\{m\}$, in the second $\psi\left(P_{1}\right) \subseteq C$; since $P_{1}$ is order connected while $C$ is an antichain, $\psi \uparrow P_{1}$ must be constant in either case. The rigid graph $(V, E)$ cannot be bipartite, so that $\psi(x)=\psi(y)$ for some $\{x, y\} \in E$; therefore $\psi(b) \in\left\{v_{6}, v_{7}\right\}$ and, consequently, $\psi\left(\operatorname{Min}\left(Q_{1}\right)\right)=\left\{v_{6}, v_{7}\right\}$ in this case. It is now easily seen that $\psi\left(Q_{1}\right) \subseteq C \cup\left\{v_{6}, v_{7}\right\}$.

The remaining possibility is that $\psi(b)=\psi(v)$ for some $v \in V$. For any $\{u, v\} \in E$ then either $\psi(b)=\psi(u)=\psi(v)$ and the previous arguments apply, or else $\operatorname{Min}(\psi(\{b, u, v\}))=\left\{v_{6}, v_{7}\right\}$. The rigidity of $(V, E)$ now implies that $\{v, w\} \in E$ for some $w \in V \backslash\{u\} ;$ if $\psi(w) \neq \psi(v)$ then $\psi(w)=\psi(u)$ must hold, reducing the argument to the 
previously considered case.

LEMMA 4.3. If $\psi$ is one-to-one on $V \cup\{b\}$ then $\psi=\Psi(\varphi)$ for some G-morphism $\varphi:\left(X_{2}, R_{2}\right)+\left(X_{1}, R_{1}\right)$.

Proof. If $\psi(x)=b$ for some $x \in V$ then also $\psi(b) \in V$. Since $(V, E)$ is a rigid graph with more than seven vertices, there exists $\{u, v\}$ not in $E$ and such that $\psi(b) \neq u, v$; by finiteness, $u=\psi(y)$ and $v=\psi(z)$ for some $y, z \in V$. By definition, there is a $q \in Q_{1}$ with $\operatorname{Min}(q)=\{x, y, z\}$, so that $\operatorname{Min}(\psi(q)\}=\psi(\operatorname{Min}(q))=\{b, u, v\}$ which contradicts the choice of $\{u, v\}$. Therefore $\psi(b)=b$ and $\psi(V)=V$.

Since $p \geq b$ and $\operatorname{Min}(p) \cap V \neq \emptyset$ if and only if $p \in E$, it follows that $\psi(E) \subseteq E$; hence $\psi$ is an endomorphism of the rigid graph $(V, E)$, that is, $\psi$ is the identity on $V \cup E$. Consequently, $\psi$ is also the identity on $C \cup\left(T \backslash\left\{t_{5}\right\}\right)$, and $\psi\left(P_{1}\right) \subseteq P_{2}$. Since, for $i<5, a_{i}$ is the only element of $P_{j}$ above $c_{i} \in C$, from $\psi\left(c_{i}\right)=c_{i}$ we obtain $\psi\left(a_{i}\right)=a_{i}$. By Proposition 2.2 there exists a $G$-morphism $\varphi:\left(X_{2}, R_{2}\right)+\left(X_{1}, R_{1}\right)$ with $\Phi(\varphi)=\psi \uparrow_{P_{1}}$. Altogether, $\psi=\Psi(\varphi)$ as was to be shown.

To complete the proof of Theorem 1.2 it suffices to note that $\Psi(\varphi)\left(Q_{1}\right)$ always represents an algebra in $B_{3} \backslash B_{2}$ while $V \cup C$ with its induced order and with the discrete topology corresponds to a finite algebra in $B_{2}$.

\section{Proof of Theorem 1.4}

The construction used and also the proof are analogous to those of the last section. Let $(V, E)$ and the set $T$ of triples be as before. Define a contravariant functor $\Psi: G \rightarrow T^{P}$ as follows.

For $(X, R) \in G$ set

$$
Q=V \cup E \cup\{b\} \cup\left(T \backslash\left\{t_{5}\right\}\right) \cup P,
$$

where the union is disjoint and $\Phi(X, R)=(P, \tau)$. The topology $\sigma$ on $Q$ again is the union of $\tau$ and the discrete topology on the finite set 
$Q \backslash P$. Conditions (i), (ii), (iii), (iv), (vi) of Section 4 together with (v') $v_{i} \leq a_{i}$ for $i<5$

define the partial order on $Q$. Set $(Q, \sigma)=\Psi(X, R)$.

For $\left(X_{i}, R_{i}\right) \in G$ and for a morphism $\varphi:\left(X_{2}, R_{2}\right) \rightarrow\left(X_{1}, R_{1}\right)$ of $G$ let $\Psi(\varphi): \Psi\left(X_{1}, R_{1}\right) \rightarrow \Psi\left(X_{2}, R_{2}\right)$ be defined as the extension of $\Phi(\varphi)$ by the identity on $Q_{1} \backslash P_{1}=Q_{2} \backslash P_{2}$, exactly as in section 4 .

Again it is routine to verify that $\Psi$ is a well defined faithful contravariant functor. Note that $\operatorname{Min}(Q)=V \cup\{b\}$ once more, while $\left|\operatorname{Min}\left(a_{i}\right)\right|=4$ for $i<5$ and $|\operatorname{Min}(q)|=3$ for all other elements of $Q \backslash \operatorname{Min}(Q)$; the space $\Psi(X, R)$ thus represents an algebra in $B_{4} \backslash B_{3}$.

Let $\psi: \Psi\left(X_{1}, R_{1}\right) \rightarrow \Psi\left(X_{2}, R_{2}\right)$ be a continuous order preserving $p$-map.

The proof of Lemma 5.1 below reads as that of Lemma 4.2 with the following modifications. First, if $\psi(u)=m=\psi(v)$ for some distinct $u, v \in V$ and if $q \in Q$ satisfies $\operatorname{Min}(q)=\{u, v, w\}$, then it is always the case that $\psi(q)=\psi(w)=m \in \operatorname{Min}\left(Q_{2}\right)$, that is, $\psi$ is constant on $V$. From $b \leq\{u, v\} \in E$ it directly follows that $\psi(b)=m$ as well. Thus $\psi \uparrow \operatorname{Min}\left(Q_{1}\right)$ is constant and, consequentiy, $\psi$ is a constant map whose value lies in $V \cup\{b\}$. If, on the other hand, $\psi(b)=\psi(v)=m$ for some $v \in V$ then, since $|\operatorname{Min}(q)| \geq 3$ for all non-minimal $q \in Q, \psi(u)=m$ whenever $\{u, v\} \in E$, so that the previous case applies.

LEMMA 5.1. If $\psi$ is not one-to-one on $V u\{b\}$ then $\psi$ is a constant with a value in $V \cup\{b\}$.

The next lemma follows along the lines of the proof of Lemma 4.3. Once it is shown that $\psi$ is the identity on $Q_{1} \backslash P_{1}=Q_{2} \backslash P_{2}$, and that $\psi\left(P_{1}\right) \subseteq P_{2}$, it suffices to note that $a_{i}$ is the only element of $P_{j}$ above $v_{i}$ in order to deduce that $\psi\left(a_{i}\right)=a_{i}$ for all $i<5$. Hence $\psi \uparrow P_{1}=\Phi(\varphi)$ as in Lemma 4.3 .

LEMMA 5.2. If $\psi$ is one-to-one on $V \cup\{b\}$ then $\psi=\Psi(\varphi)$ for 
some compatible $\varphi:\left(X_{2}, R_{2}\right) \rightarrow\left(X_{1}, R_{1}\right)$.

The space $\Psi(\varphi)\left(Q_{1}\right)$ represents an algebra in $B_{4} \backslash B_{3}$ for every compatible $\varphi$, while any other map $\psi$ is constant; thus its image represents one of the finitely many maps whose image represents a twoelement Boolean subalgebra of $\Psi\left(X_{1}, R_{1}\right)$. This concludes the proof of Theorem 1.4 .

\section{References}

[1] M.E. Adams, V. Koubek and J. Sichler, "Homomorphisms and endomorphisms in varieties of pseudocomplemented distributive lattices (with applications to Heyting algebras)", submitted.

[2] M.E. Adams, V. Koubek and J. Sichler, "Homomorphisms and endomorphisms of distributive lattices", submitted.

[3] R. Balbes and Ph. Dwinger, Distributive lattices (University of Missouri Press, Columbia, Missouri, 1974).

[4] B.A. Davey and D. Duffus, "Exponentiation and duality", Ordered sets, 43-95 (Reidel, New York, 1982).

[5] G. Grätzer, Lattice theory: first concepts and distributive lattices (Freeman, San Francisco, California, 1971).

[6] G. Grätzer, General lattice theory (Birkhauser Verlag; Basel and Stuttgart, 1978).

[7] G. Grätzer and H. Lakser, "The structure of pseudocomplemented distributive lattices II: congruence extension and amalgamation", Trans. Amer. Math. Soc. 156 (1971), 343-358.

[8] G. Grätzer and H. Lakser, "The structure of pseudocomplemented distributive lattices III: injective and absolute subretracts", Trans. Amer. Math. Soc. 169 (1972), 475-487.

[9] Z. Hedrlín and A. Pultr, "Relations (graphs) with given infinite semigroup", Monatsh. Math. 68 (1964), 421-425.

[10] H. Lakser, "The structure of pseudocomplemented distributive lattices I", Trans. Amer. Math. Soc. 156 (1971), 335-342. 
[11] K.B. Lee, "Equational classes of distributive pseudocomplemented lattices", Canad. J. Math. 22 (1970), 881-891.

[12] K.D. Magill, "The semigroup of endomorphisms of a Boolean ring", Semigroup Forum 4 (1972), 411-416.

[13] H.A. Priestley, "Representation of distributive lattices by means of ordered Stone spaces", Bull. Lordon Math. Soc. 2 (1970), $186-190$.

[14] H.A. Priestley, "Ordered topological spaces and the representation of distributive lattices", Proc. London Math. Soc. (3) 24 (1972), 507-530.

[15] H.A. Priestley, "The construction of spaces dual to pseudocomplemented distributive lattices", Quart. J. Math. Oxford (2) 26 (1975), 215-228.

[16] H.A. Priestley, "Ordered sets and duality for distributive lattices", Proc. Conf. on Ordered Sets and their Applications, Lyon, 1982 (to appear).

[17] A. Puitr and V. Trnková, Combinatorial, algebraic and topological representations of groups, semigroups and categomies (NorthHolland, Amsterdam, 1980).

[18] P. Ribenboim, "Characterization of the sup-complement in a distributive lattice with last element", Summa Brasil. Math. 2 (1949), 43-49.

[19] B.M. Schein, "Ordered sets, semilattices, distributive lattices and Boolean algebras with homomorphic endomorphism semigroups", Fund. Math. 68 (1970), 31-50.

Department of Mathematics, State University of New York, New Paltz, New York 12561,

USA;

Department of Mathematics and Astronomy, University of Manitoba, Winnipeg, Manitoba,

Canada R3T 2N2.
MFF KU Malostranské nám. 25, Praha 1, Czechos lovakia; 